\title{
Entanglement of quantum oscillators coupled to different heat baths
}

\author{
Wei-Can Syu, Da-Shin Lee, Chen-Pin Yeh \\ Department of Physics, National Dong Hwa University, Hualien 97401, Taiwan, R.O.C. \\ E-mail: syuweican@gmail.com
}

\begin{abstract}
We study the non-equilibrium dynamics of two coupled oscillators interacting with their own heat baths of quantum scalar fields at different temperature $T_{1}$ and $T_{2}$ with bilinear couplings between them. We particularly focus on the entanglement or inseparability property of their quantum states. The critical temperatures of two respective oscillators, $T_{1 c}$ and $T_{2 c}$, higher than which the entanglement disappears, can be determined. It is found that when two damping parameters are largely different, say $\gamma_{1} \ll \gamma_{2}$, the critical temperature $T_{1 c}$ with respect to the frequency $\Omega_{+}$, the higher frequency among two normal modes frequencies, can be very large, $T_{1 c} \gg \Omega_{+}$, while $T_{2 c} \propto \Omega_{+}$with the possibility of hot entanglement. The entanglement of two oscillators with the temperature-dependent damping parameters $\gamma_{1 ; 2, T}$ from heat baths is also discussed.
\end{abstract}

PACS numbers: 03.65.Ud, 03.65.Yz, 03.67.-a 


\section{Introduction}

Entanglement or inseparability of composite quantum systems is a characteristic trait of quantum mechanics lacking in classical mechanics, and then becomes a key resource in many quantum information processing protocols. The degree of entanglement or mixture is the central concern from the viewpoints of quantum information and quantum computation. Thus, it is of great importance to search for a proper mathematical frameworks to quantify such features in general mixed quantum states. A step toward this goal is to find the criterions to distinguish between quantum and classical correlations of mixed states. A bipartite state is said to be separable if the total density matrix $\rho_{A B}$ can be written in the form, $\rho_{A B}=\sum_{i} p_{i}\left(\left|\phi_{i}\right\rangle\left\langle\phi_{i}\right|\right)_{A} \otimes\left(\left|\psi_{i}\right\rangle\left\langle\psi_{i}\right|\right)_{B}$ with $p_{i}>0$. Two subsystems $A$ and $B$ are said to be quantum entangled if they are not separable. One criterion of the separability was proposed by Peres [1] and later further extended by Horodecki $[2,3]$ in the case of discrete variable quantum systems in finite-dimensional Hilbert space. The application of PeresHorodecki criterions to continuous variable systems in infinite-dimensional Hilbert space [4] has also received much attention. Some other entanglement measures such as negativity [5] and logarithmic negativity $[6,7,8]$ are also suggested for general bipartite states.

The quantum-mechanical degrees of freedom unavoidably couple to environments or baths, which induce dissipation and decoherence. The effective theory can be described by the reduced density matrix, which is obtained by tracing out environmental variables in the full density matrix using the method of Feyman-Vernon influence functional [9], and becomes an essential quantity for constructing the entanglement measures mentioned above. This concept has been used in pioneering works on quantum Brownian motion and general open quantum systems $[10,11,12]$. Recently there have been remarkable experiments $[13,14,15,16]$ using an opto-mechanical resonator to engineer the properties of the environmental degrees of freedom and its coupling to the probed systems, which make it possible to couple with several different baths, and to tailor decoherence and dissipative properties in such an experimentally controlling manner. Thus, the experimental advances motivate theoretical studies, showing that coupling to different non-equilibrium environments can lead to the persistence of entanglement in the high temperature limit $[17,18,19]$. The idea is to consider the model of two coupled, parametrically driven, dissipative harmonic oscillators with the typical energy scale $E$, which couple to its own heat bath at the same temperature $T$. It is expected that when $k_{B} T>E$, the quantum nature of the system such as superposition and entanglement will be lost. However, it was argued that the nonequilibrium dynamics can bring the system into the late-time saturation characterized by an effective temperature, which is the relevant scale for determining whether or not quantum features of the system can survive [19]. The effective temperature can be much lower than the temperature of the bath $T$ so as to sustain quantum entanglement even at high temperature $T$ [19]. The works of $[20,21]$ reexamine the above-mentioned model by considering time-independent couplings between two oscillators with the same Ohmic damping parameters, $\gamma_{1}=\gamma_{2}$. Additionally the temperatures of two baths are set to be 
different, say $T_{1}$ and $T_{2}$ respectively. The late-time nonequilibrium steady states are found where the critical temperature below which quantum entanglement exists turns out not so surprisingly to be $k_{B} T_{c} \sim E \sim \Omega_{+}$where $\Omega_{+}$is the higher frequency among two normal modes frequencies. It seems that parametric driving with the time-dependent coupling between two oscillators is a key mechanism for sustaining the entanglement in the system at high temperature. Nevertheless, in [22], coherence of two mechanical oscillators coupled to different and uncorrelated baths is studied, and is found to be enhanced in the case of $\gamma_{1} \neq \gamma_{2}$. Thus, in this work we plan to extend the works of $[20,21]$ by considering the case when $\gamma_{1} \neq \gamma_{2}$ with the hope to boost the critical temperature for the entanglement to a higher one as compared with the case of $\gamma_{1}=\gamma_{2}$. Recently, the model above has also been adopted to successfully explain the experimental demonstration of a novel effect of heat transfer between two objects driven by quantum vacuum fluctuations [23]. Theoretic studies of this model on heat transfer between microsystems are also found for example in $[24,25,26,27]$.

Our presentation is organized as follows. In next section, we introduce the model, and briefly review the idea of open quantum system with the method of the closedtime-path formalism. The environmental degrees of freedom in the full density matrix of the system-plus-environment are traced over to obtain the reduced density matrix of the system. Environmental effects are then all encoded in the influence functional, which can be determined by nonequilibrium two-point correlators. Then the corresponding HeisenbergLangevin equations for two oscillators involving the damping as well as noise terms are derived, which can be solved for the general solutions. In Sec. 3, we construct the covariance matrix, and substitute the solutions of the Heisenberg-Langevin equations into the matrix elements. In the late-time limit, the matrix elements are dominated by the noise correlations on the oscillators. The separability criterions are discussed. Violation of the criterions allows us to compute the critical temperature of the entanglement both numerically and analytically in Sec. 4. We summarize all results with their implications in Sec. 6.

\section{The model: two oscillators coupled to its own heat baths}

We consider the model of two quantum oscillators coupled to their own heat baths of quantum scalar fields at different temperatures. The action is described by $[28,20,21]$

$$
\begin{aligned}
S[\chi, \varphi]=S_{\chi}\left[\chi_{1}, \chi_{2}\right]+S_{\varphi}\left[\varphi_{1}, \varphi_{2}\right]+S_{\chi \varphi}\left[\chi_{1,2}, \varphi_{1,2}\right] \\
=\int d s\left[\frac{m}{2}\left(\dot{\chi}_{1}\right)^{2}-\frac{m \Omega^{2}}{2} \chi_{1}^{2}+\frac{m}{2}\left(\dot{\chi}_{2}\right)^{2}-\frac{m \Omega^{2}}{2} \chi_{2}^{2}-m \sigma \chi_{1}(s) \chi_{2}(s)\right] \\
+\int d^{4} x \frac{1}{2}\left[\partial_{\mu} \varphi_{1} \partial^{\mu} \varphi_{1}+\partial_{\mu} \varphi_{2} \partial^{\mu} \varphi_{2}\right] \\
+g_{1} \int d^{4} x \chi_{1}(s) \delta^{(3)}\left(\mathbf{x}-\mathbf{z}_{1}(s)\right) \varphi_{1}(x) \\
+g_{2} \int d^{4} x \chi_{2}(s) \delta^{(3)}\left(\mathbf{x}-\mathbf{z}_{2}(s)\right) \varphi_{2}(x) .
\end{aligned}
$$


Two oscillators have the same oscillation frequency $\Omega$ and mass $m$, and they couple to each other with a coupling constant $\sigma$. The coupling of the oscillator $\chi_{1,2}$ to the heat bath of a scalar field $\varphi_{1,2}$ has the coupling strength $g_{1,2}$. Moreover, $\mathbf{z}_{1,2}$ is the prescribed trajectory of the oscillators. Here we assume that the initial density matrix for the whole system (oscillators-plus-fields) at time $t_{i}=0$ is factorizable as

$$
\rho(0)=\rho_{\chi}(0) \otimes \rho_{\varphi_{1}} \otimes \rho_{\varphi_{2}} .
$$

The fields are initially in thermal equilibrium at temperature $\beta_{1}=1 / T_{1}$ for the field $\varphi_{1}$ and $\beta_{2}=1 / T_{2}$ for the field $\varphi_{2}$ with $T_{1} \neq T_{2}$ in general. Their respective density matrices are given by

$$
\rho_{\varphi_{1}}=e^{-\beta_{1} H\left[\varphi_{1}\right]} / \operatorname{Tr}\left\{e^{-\beta_{1} H\left[\varphi_{1}\right]}\right\}, \quad \rho_{\varphi_{2}}=e^{-\beta_{2} H\left[\varphi_{2}\right]} / \operatorname{Tr}\left\{e^{-\beta_{2} H\left[\varphi_{2}\right]}\right\},
$$

where $H\left[\varphi_{1,2}\right]$ is the Hamiltonian for the free field $\varphi_{1,2}$, constructed from $S_{\varphi}\left[\varphi_{1}, \varphi_{2}\right]$ in (1). The density matrix $\rho(t)$ of the whole system evolves unitarily according to

$$
\rho(t)=U(t, 0) \rho(0) U^{-1}(t, 0),
$$

where $U(t, 0)$ is the time evolution operator. The effects from the environment to the system can be summarized in the reduced density matrix $\rho_{r}(t)$, which is obtained by tracing out the environmental degrees of freedom in $\rho(t)$. Here we provide a brief summary of the main results of the reduced density matrix of the system, and a mini review of how to obtain the equations of motion for the position operators of two coupled oscillators with environmental effects. We start with the reduced density matrix, expressed as

$$
\begin{aligned}
& \rho_{\chi}\left(\chi_{1 F}, \chi_{1 F}^{\prime}, \chi_{2 F}, \chi_{2 F}^{\prime} ; t\right)=\int_{-\infty}^{\infty}\left\{\prod_{a=1}^{2} d \chi_{a I} d \chi_{a I}^{\prime}\right\} \rho_{\chi}\left(\chi_{1 I}, \chi_{1 I}^{\prime}, \chi_{2 I}, \chi_{2 I}^{\prime}, 0\right) \\
& \left\{\prod_{a=1}^{2} \int_{\chi_{a I}}^{\chi_{a F}} \mathcal{D} \chi_{a+} \int_{\chi_{a I}^{\prime}}^{\chi_{a F}^{\prime}} \mathcal{D} \chi_{a-}\right\} \exp \left(i S_{\chi}\left[\chi_{1+}, \chi_{2+}\right]-i S_{\chi}\left[\chi_{1-}, \chi_{2-}\right]\right) \times \mathcal{F}\left[\chi_{1+}, \chi_{2+}, \chi_{1-}, \chi_{2-}\right] .
\end{aligned}
$$

The influence functional $\mathcal{F}$ can be written in terms of nonequilibrium two-point correlators constructed from the environment fields as

$$
\begin{aligned}
& \mathcal{F}\left[\chi_{1+}, \chi_{2+}, \chi_{1-}, \chi_{2-}\right]=e^{i S_{I F}\left[\chi_{1+}, \chi_{2+}, \chi_{1-}, \chi_{2-}\right]} \\
& =\prod_{a=1}^{2} \exp \left\{\frac { i } { 2 } \int _ { 0 } ^ { t } d s d s ^ { \prime } \left(\left[\chi_{a+}(s)-\chi_{a-}(s)\right] G_{R, T_{a}}\left(s, s^{\prime}\right)\left[\chi_{a+}\left(s^{\prime}\right)+\chi_{a-}\left(s^{\prime}\right)\right]\right.\right. \\
& \left.\left.\quad+i\left[\chi_{a+}(s)-\chi_{a-}(s)\right] G_{H, T_{a}}\left(s, s^{\prime}\right)\left[\chi_{a+}\left(s^{\prime}\right)-\chi_{a-}\left(s^{\prime}\right)\right]\right)\right\}
\end{aligned}
$$

where $S_{I F}$ is called the influence action. The retarded Green's function $G_{R, T_{a}}$ is defined by

$$
G_{R, T_{a}}\left(s, s^{\prime}\right)=i g_{a}^{2} \theta\left(s-s^{\prime}\right)\left\langle\left[\varphi_{a}\left(\mathbf{z}_{a}(s), s\right), \varphi_{a}\left(\mathbf{z}_{a}\left(s^{\prime}\right), s^{\prime}\right)\right]\right\rangle
$$


and the Hadamard function $G_{H, T_{a}}$ by

$$
G_{H, T_{a}}\left(s, s^{\prime}\right)=\frac{g_{a}^{2}}{2}\left\langle\left\{\varphi_{a}\left(\mathbf{z}_{a}(s), s\right), \varphi_{a}\left(\mathbf{z}_{a}\left(s^{\prime}\right), s^{\prime}\right)\right\}\right\rangle
$$

with $a=1,2$. Notice that we have absorbed the coupling constant $g_{a}^{2}$ into the definition of the Green's functions. The Hadamard function is simply the expectation value of the anticommutator of the quantum field $\varphi_{a}$ with respect to the thermal state at temperature $T_{a}$, and notice that the retarded Green's function does not have any temperature dependence due to the linear coupling of the scalar field to the oscillator. The kernels $G_{H, T_{a}}$ and $G_{R, T_{a}}$ respectively are in turn linked by the fluctuation-dissipation relation. The fluctuationdissipation relation is known to play a pivotal role in balancing these two effects in order to dynamically stabilize the nonequilibrium evolution of the system under a fluctuating environment. Mathematically, it relates the Fourier transform of the fluctuation kernel $G_{H, T_{a}}$ to the imaginary part of the retarded kernel $G_{R, T_{a}}$ as follows

$$
\begin{aligned}
& G\left(s, s^{\prime}\right)=\int \frac{d \omega}{2 \pi} G\left(\mathbf{z}(s), \mathbf{z}\left(s^{\prime}\right) ; \omega\right) e^{-i \omega\left(s-s^{\prime}\right)} \\
& G_{H, T_{a}}\left(\mathbf{z}(s), \mathbf{z}\left(s^{\prime}\right) ; \omega\right)=\operatorname{coth}\left[\frac{\omega}{2 T_{a}}\right] \operatorname{Im} G_{R, T_{a}}\left(\mathbf{z}(s), \mathbf{z}\left(s^{\prime}\right) ; \omega\right) .
\end{aligned}
$$

Thus, one can define the coarse-grained effective action as

$$
S_{C G}\left[\chi_{1+}, \chi_{2+}, \chi_{1-}, \chi_{2-}\right]=S_{\chi}\left[\chi_{1+}, \chi_{2+}\right]-S_{\chi}\left[\chi_{1-}, \chi_{2-}\right]+S_{I F}\left[\chi_{1+}, \chi_{2+}, \chi_{1-}, \chi_{2-}\right] .
$$

To find the time evolution of two oscillators, we derive the Heisenberg-Langevin equations for the quantum operators $\hat{\chi}_{1}$ and $\hat{\chi}_{2}$ that incorporate environmental effects. This can be done by introducing an auxiliary variable $\eta_{T_{a}}(s)$, the noise force, with a Gaussian distribution function:

$$
P\left[\eta_{T_{a}}(s)\right]=\exp \left\{-\frac{1}{2} \int_{0}^{t} d s \int_{0}^{t} d s^{\prime} \eta_{T_{a}}(s) G_{H, T_{a}}^{-1}\left(s-s^{\prime}\right) \eta_{T_{a}}\left(s^{\prime}\right)\right\} .
$$

In terms of the noise force $\eta_{T_{a}}(s), S_{C G}$ can be rewritten as an ensemble average over $\eta_{T_{a}}(s)$,

$$
\exp i S_{C G}\left[\chi_{1+}, \chi_{2+}, \chi_{1-}, \chi_{2-}\right]=\int \prod_{a=1}^{2} \mathcal{D} \eta_{T_{a}} P\left[\eta_{T_{a}}(s)\right] \exp i S_{\eta}\left[\chi_{1+}, \chi_{2+}, \chi_{1-}, \chi_{2-} ; \eta_{1}, \eta_{2}\right]
$$

where the stochastic coarse-grained effective action $S_{\eta}$ is given by

$$
\begin{aligned}
& S_{\eta}\left[\chi_{1+}, \chi_{2+}, \chi_{1-}, \chi_{2-} ; \eta_{1}, \eta_{2}\right]=S_{\chi}\left[\chi_{1+}, \chi_{2+}\right]-S_{\chi}\left[\chi_{1-}, \chi_{2-}\right] \\
&+\sum_{a=1}^{2} \frac{1}{2} \int_{0}^{t} d s \int_{0}^{t} d s^{\prime}\left[\chi_{a+}(s)-\chi_{a-}(s)\right] G_{R, T_{a}}\left(s, s^{\prime}\right)\left[\chi_{a+}\left(s^{\prime}\right)+\chi_{a-}\left(s^{\prime}\right)\right] \\
&+\sum_{a=1}^{2} \int_{0}^{t} d s \eta_{T_{a}}(s)\left[\chi_{a+}(s)-\chi_{a-}(s)\right]
\end{aligned}
$$

Then, the equations of motion for $\hat{\chi}_{1}$ and $\hat{\chi}_{2}$ can be derived from the action $S_{\eta}$ by introducing the center of mass coordinate $\chi_{a}$ and the relative coordinate $R_{a}, \chi_{a}=\frac{1}{2}\left(\chi_{a+}+\chi_{a-}\right), R_{a}=$ 
$\chi_{a+}-\chi_{a-}$, and then taking the variation of $S_{\eta}$ with respect to $R_{a}$. For simplicity, we assume that two oscillators barely move away from the spatial locations of $\mathbf{z}_{0 a}$ so that $\mathbf{z}_{a}(s) \simeq \mathbf{z}_{0 a}$. In this approximation, the retarded Green's function $G_{R, T_{a}}$ and the Hadamard function $G_{H, T_{a}}$ can be expressed as

$$
\begin{aligned}
& G_{R, T_{a}}\left(s, s^{\prime}\right)=-\frac{1}{2 \pi} g_{a}^{2} \theta\left(s-s^{\prime}\right) \delta^{\prime}\left(s-s^{\prime}\right), \\
& G_{H, T_{a}}\left(s, s^{\prime}\right)=\frac{g_{a}^{2}}{4 \pi} \int \frac{d \omega}{2 \pi} \omega \operatorname{coth}\left[\frac{\omega}{2 T_{a}}\right] e^{-i \omega\left(s-s^{\prime}\right)} .
\end{aligned}
$$

With the Green's functions above, the Heisenberg-Langevin equations for quantum oscillators can be written as

$$
\begin{aligned}
& \ddot{\hat{\chi}}_{1}+2 \gamma_{1} \dot{\hat{\chi}}_{1}+\Omega^{2} \hat{\chi}_{1}+\sigma \hat{\chi}_{2}=\frac{\eta_{T_{1}}}{m}, \\
& \ddot{\hat{\chi}}_{2}+2 \gamma_{2} \dot{\hat{\chi}}_{2}+\Omega^{2} \hat{\chi}_{2}+\sigma \hat{\chi}_{1}=\frac{\eta_{T_{2}}}{m} .
\end{aligned}
$$

The effects from the retarded Green's function $G_{R a}$ not only slow down the motion of $\chi$ through the induced damping terms with the damping parameters $\gamma_{a}=g_{a}^{2} / 8 \pi m$, but also give a shift to the oscillation frequency, with $\delta \Omega_{a}^{2}=-4 \gamma_{a} \delta(0)$. Here we consider the weak oscillator-bath couplings with small $g_{a}$ where the corrections $\delta \Omega_{a}^{2}$ can be ignored as compared with $\Omega^{2}$.

It worths mentioning that there are two different sources of fluctuations, over which the averages are taken on $\hat{\chi}_{1}$ and $\hat{\chi}_{2}$. One is the average over intrinsic quantum fluctuations of the oscillators, and the other is the average over the noise manifested from thermal fluctuations of the environments. The distribution function $P\left[\eta_{T_{a}}(s)\right]$ in (10) leads to the correlation function of the noise as follows

$$
\left\langle\eta_{T_{a}}(s) \eta_{T_{a}}\left(s^{\prime}\right)\right\rangle=G_{H, T_{a}}\left(s-s^{\prime}\right),
$$

where

$$
G_{H, T_{a}}\left(s-s^{\prime}\right)=2 m \gamma_{a} \int \frac{d \omega}{2 \pi} \omega \operatorname{coth}\left[\frac{\omega}{2 T_{a}}\right] e^{-i \omega\left(s-s^{\prime}\right)}
$$

In [22], the model of (1) has been considered where the environment consists of a collection of harmonic oscillators. The Heisenberg equations of motion of the system and the environment can be solved exactly, giving the same form of the equations in (14) and (15) with the Green's functions constructed out of the harmonic oscillators. In this work, we derive the Heisenberg-Langevin equations from the influence functional.

\section{Covariance matrix and separability criterions}

The initial states of two quantum oscillators are Gaussian wave packets with the initial conditions on the expectation values of the position operator $\hat{\chi}_{a}$ and the momentum operator 
$\hat{p}_{a}=m \dot{\hat{\chi}}_{a}$ as follows

$$
\begin{array}{ll}
\left\langle\hat{\chi}_{a}(0)\right\rangle & =\left\langle\hat{p}_{a}(0)\right\rangle=0, \quad\left\langle\left\{\hat{\chi}_{a}(0), \hat{p}_{b}(0)\right\}\right\rangle=0, \\
\left\langle\left\{\hat{\chi}_{a}(0), \hat{\chi}_{b}(0)\right\}\right\rangle=\delta_{a b}\left\langle\hat{\chi}_{a}^{2}(0)\right\rangle, & \left\langle\left\{\hat{p}_{a}(0), \hat{p}_{b}(0)\right\}\right\rangle=\delta_{a b}\left\langle\hat{p}_{a}^{2}(0)\right\rangle,
\end{array}
$$

where two oscillators are initially in a separable state. The interaction between two oscillators starts to build up the entanglement between them.

A Gaussian state is completely characterized by its first and second statistical moments of a raw matrix defined as $\hat{\mathbf{X}}=\left(\hat{\chi}_{1}, \hat{p}_{1}, \hat{\chi}_{2}, \hat{p}_{2}\right)$, where the first moments vanish, $\langle\hat{\mathbf{X}}\rangle=0$ due to the chosen initial conditions and the second moments are specified by the covariance matrix given by

$$
V_{i j}=\frac{1}{2}\left\langle\hat{X}_{i} \hat{X}_{j}+\hat{X}_{j} \hat{X}_{i}\right\rangle-\left\langle\hat{X}_{i}\right\rangle\left\langle\hat{X}_{j}\right\rangle
$$

with $i, j=1,2,3,4$. For any operator $\hat{O},\langle\hat{O}\rangle=\operatorname{Tr}\left[\rho_{\chi} \hat{O}\right]$ where $\rho_{\chi}$ is the reduced density matric of $\chi_{1}$ and $\chi_{2}$. Apparently, $\mathbf{V}$ is a real symmetric matrix, namely $\mathbf{V}=\mathbf{V}^{T}$. Also, with the Gaussian states described above, we have $V_{23}=-V_{14}, V_{31}=V_{13}, V_{32}=-V_{41}$, $V_{41}=-V_{14}, V_{42}=V_{24}$ and $V_{12}=V_{21}=V_{34}=V_{43}=0$ where the covariance matrix can be written as

$$
\mathbf{V}=\left(\begin{array}{cc}
A & C \\
C^{T} & B
\end{array}\right)
$$

with

$$
A=\left(\begin{array}{cc}
V_{11} & 0 \\
0 & V_{22}
\end{array}\right), \quad B=\left(\begin{array}{cc}
V_{33} & 0 \\
0 & V_{44}
\end{array}\right), \quad \text { and } C=\left(\begin{array}{cc}
V_{13} & V_{14} \\
-V_{14} & V_{24}
\end{array}\right)
$$

Considering the positive definite density matrix $\rho_{\chi}$, Heisenberg uncertainty principles can be cast in the form $[29,30]$

$$
\mathbf{V}+i \mathbf{K} \geq 0
$$

with the matrix $\mathbf{K}$ defined as

$$
\mathbf{K}=\left(\begin{array}{cccc}
0 & 1 & 0 & 0 \\
-1 & 0 & 0 & 0 \\
0 & 0 & 0 & 1 \\
0 & 0 & -1 & 0
\end{array}\right)
$$

and should be obeyed by any quantum system. The uncertainty principle is a direct consequence of the canonical commutation relation and the non-negativity of the density matrix. Moreover $\mathbf{V}$ can be diagonalized by the sympletic transformation $S \in S p(4, R)$, the ten-parameter real symplectic group, where two sympletic eigenvalues, $\eta_{\lessgtr}$ (with $\eta_{>}>\eta_{<}$) would satisfy $\eta_{\lessgtr} \geq 1 / 2$ as the result of (23) and encode essential information for the Gaussian states [4]. According to [1,2], one can define the partial transpose $\bar{\rho}_{\chi}$ of a bi-particle quantum state $\rho_{\chi}$ as the transpose performed on one of two particles in a given basis. Accordingly, 
the the partial transpose covariance matrix is denoted as $\overline{\mathbf{V}}$. Together with the positivity of the partially transposed density matrix $\bar{\rho}_{\chi}$, the necessary and sufficient separability criterion (Peres-Horodecki-Simon (PHS) criterion) for bipartite continuous Gaussian variable systems is then expressed as [4]

$$
\overline{\mathbf{V}}+i \mathbf{K} \geq 0 .
$$

The violation of the PHS criterion indicates the existence of quantum entanglement between two Gaussian systems. Again, the associated two sympletic eigenvalues $\bar{\eta}_{\lessgtr}$ of $\overline{\mathbf{V}}$ would satisfy $\bar{\eta}_{\lessgtr} \geq 1 / 2$ due to $(25)$. The expressions of $\bar{\eta}_{\lessgtr}$ in terms of the matrix $A, B$, and $C$ defined above will be discussed below as a measure of quantum entanglement of the Gaussian states in this study.

To find the covariance matrix we need to solve Eqs.(14) and (15) where the general solutions are the sum of the homogeneous solution $\chi_{a ; h}$ that depends on the initial conditions, $\chi_{a}(0)$ and $\dot{\chi}_{a}(0)$, and the particular solution $\chi_{a ; p}$ that satisfies the full equations with the noise. Let us write the time dependence of $\chi_{a ; h}$ as $\chi_{a ; h}(t) \propto e^{-i \bar{\Omega}_{a, \pm} t}$. The frequency $\bar{\Omega}_{a, \pm}$ is determined by the equation, $\tilde{\mathbf{D}}^{-1}\left(\bar{\Omega}_{a, \pm}\right)=0$ with the matrix $\tilde{\mathbf{D}}(\omega)$ defined as

$$
\tilde{\mathbf{D}}(\omega)=\frac{1}{-\omega^{2} \mathbf{I}+\Omega^{2}-i 2 \omega \boldsymbol{\Gamma}}
$$

where

$$
\mathbf{I}=\left(\begin{array}{ll}
1 & 0 \\
0 & 1
\end{array}\right), \quad \boldsymbol{\Omega}^{2}=\left(\begin{array}{cc}
\Omega^{2} & \sigma \\
\sigma & \Omega^{2}
\end{array}\right), \quad \boldsymbol{\Gamma}=\left(\begin{array}{cc}
\gamma_{1} & 0 \\
0 & \gamma_{2}
\end{array}\right)
$$

This gives the following determinant,

$$
\operatorname{det} \tilde{D}^{-1}(\omega)=\left[\left(-\omega^{2}+\Omega^{2}-i 2 \omega \gamma_{1}\right)\left(-\omega^{2}+\Omega^{2}-i 2 \omega \gamma_{2}\right)-\sigma^{2}\right]
$$

Solving $\tilde{\mathbf{D}}^{-1}\left(\bar{\Omega}_{a, \pm}\right)=0$, we find

$$
\begin{aligned}
& \bar{\Omega}_{1, \pm} \simeq \Omega_{+} \pm \frac{i}{2}\left(\gamma_{1}+\gamma_{2}\right), \\
& \bar{\Omega}_{2, \pm} \simeq \Omega_{-} \pm \frac{i}{2}\left(\gamma_{1}+\gamma_{2}\right),
\end{aligned}
$$

with $\Omega_{+} \equiv \sqrt{\Omega^{2}+\sigma}, \Omega_{-} \equiv \sqrt{\Omega^{2}-\sigma}$.

We consider that two oscillators undergo underdamped oscillations in the case of $\Omega^{2}>\sigma$ and also both $\Omega$ and $\sqrt{\sigma}$ being much larger than $\gamma_{1}$ and $\gamma_{2}$. Asymptotically as $t \rightarrow \infty$, the homogeneous solution $\chi_{a ; h}$ damps out exponentially and its contributions to the covariance matrix with the initial condition dependence can be ignored. Thus, the late-time behavior of the covariance matrix is determined by a particular solution $\chi_{a ; p}$ due to the noise terms 
$\eta_{T_{a}}$, which then lead to the following non-vanishing elements

$$
\begin{aligned}
V_{11} & =\frac{1}{2}\left\langle\chi_{1}(\infty), \chi_{1}(\infty)\right\rangle \\
& =\frac{1}{m^{2}} \int_{-\infty}^{\infty} \frac{d \omega}{2 \pi}\left(\left|\tilde{D}_{11}(\omega)\right|^{2} \tilde{G}_{H, T_{1}}(\omega)+\left|\tilde{D}_{12}(\omega)\right|^{2} \tilde{G}_{H, T_{2}}(\omega)\right) \\
V_{22} & =\frac{1}{2}\left\langle p_{1}(\infty), p_{1}(\infty)\right\rangle \\
& =\int_{-\infty}^{\infty} \frac{d \omega}{2 \pi} \omega^{2}\left(\left|\tilde{D}_{11}(\omega)\right|^{2} \tilde{G}_{H, T_{1}}(\omega)+\left|\tilde{D}_{12}(\omega)\right|^{2} \tilde{G}_{H, T_{2}}(\omega)\right) \\
V_{33} & =\frac{1}{2}\left\langle\chi_{2}(\infty), \chi_{2}(\infty)\right\rangle \\
& =\frac{1}{m^{2}} \int_{-\infty}^{\infty} \frac{d \omega}{2 \pi}\left(\left|\tilde{D}_{21}(\omega)\right|^{2} \tilde{G}_{H, T_{1}}(\omega)+\left|\tilde{D}_{22}(\omega)\right|^{2} \tilde{G}_{H, T_{2}}(\omega)\right) \\
V_{44} & =\frac{1}{2}\left\langle p_{2}(\infty), p_{2}(\infty)\right\rangle \\
& =\int_{-\infty}^{\infty} \frac{d \omega}{2 \pi} \omega^{2}\left(\left|\tilde{D}_{21}(\omega)\right|^{2} \tilde{G}_{H, T_{1}}(\omega)+\left|\tilde{D}_{22}(\omega)\right|^{2} \tilde{G}_{H, T_{2}}(\omega)\right) \\
V_{13} & =\frac{1}{2}\left\langle\chi_{1}(\infty), \chi_{2}(\infty)\right\rangle \\
& =\frac{1}{m^{2}} \int_{-\infty}^{\infty} \frac{d \omega}{2 \pi}\left(\tilde{D}_{11}^{*}(\omega) \tilde{D}_{21}(\omega) \tilde{G}_{H, T_{1}}(\omega)+\tilde{D}_{12}^{*}(\omega) \tilde{D}_{22}(\omega) \tilde{G}_{H, T_{2}}(\omega)\right) \\
V_{14} & =\frac{1}{2}\left\langle\chi_{1}(\infty), p_{2}(\infty)\right\rangle \\
& =\frac{-i}{m} \int_{-\infty}^{\infty} \frac{d \omega}{2 \pi} \omega\left(\tilde{D}_{11}^{*}(\omega) \tilde{D}_{21}(\omega) \tilde{G}_{H, T_{1}}(\omega)+\tilde{D}_{12}^{*}(\omega) \tilde{D}_{22}(\omega) \tilde{G}_{H, T_{2}}(\omega)\right) \\
V_{24} & =\frac{1}{2}\left\langle p_{1}(\infty), p_{2}(\infty)\right\rangle \\
& \int_{-\infty}^{\infty} \frac{d \omega}{2 \pi} \omega^{2}\left(\tilde{D}_{11}^{*}(\omega) \tilde{D}_{21}(\omega) \tilde{G}_{H, T_{1}}(\omega)+\tilde{D}_{12}^{*}(\omega) \tilde{D}_{22}(\omega) \tilde{G}_{H, T_{2}}(\omega)\right) \\
& \\
&
\end{aligned}
$$

In the case of continuous Gaussian variables, it has been shown that PHS separability criterion in (25) in quantum systems together with the Heisenberg uncertainty relation in (23) is equivalent to the following inequalities, expressed in terms of the $S p(2, R) \otimes S p(2, R)$ invariants constructed out of the matrices $A, B$, and $C$ in (22) as [4]

$\zeta_{ \pm}=\operatorname{det} A \cdot \operatorname{det} B-\operatorname{Tr}\left(A \cdot J \cdot C \cdot J \cdot B \cdot J \cdot C^{T} \cdot J\right)-\frac{1}{4}(\operatorname{det} A+\operatorname{det} B)+\left(\operatorname{det} C \pm \frac{1}{4}\right)^{2} \geq 0$.

where the matrix $J$ is defined to be

$$
J=\left(\begin{array}{cc}
0 & 1 \\
-1 & 0
\end{array}\right)
$$


In this form, the condition for $\zeta_{-}$is the Heisenberg uncertainty relation while the condition for $\zeta_{+}$is the PHS criterion, as can be noticed from (23) and (25). In fact, the change from the density matrix $\rho_{\chi}$ to the partial transposed density matrix $\bar{\rho}_{\chi}$ gives the sign flip in $\operatorname{det} C$. Because $\zeta_{+}=\zeta_{-}+\operatorname{det} C$, the assumption that the uncertainty relation, $\zeta_{-} \geq 0$ always true leads to $\zeta_{+} \geq 0$ when $\operatorname{det} C>0$. For $\operatorname{det} C<0$, the possibility of $\zeta_{+}<0$ implies the existence of quantum entanglement [4]. So, the condition, $\operatorname{det} C=V_{13} V_{24}+V_{14}^{2}<0$, indicates the possible existence of quantum entanglement in the bipartite Gaussian states, which highlights the importance of cross-correlations on the entanglements, giving $\zeta_{+}<0$.

Once we have the covariance matrix for the coupled oscillators in nonequilibrium steady states, we construct $\zeta_{+}$according to (38) [20, 21]. In fact, $\overline{\mathbf{V}}$ can be diagonalized by the sympletic transformation $S \in S p(4, R)$. In particular, the sympletic eigenvalues of partial transport $\overline{\mathbf{V}}$ are

$$
\bar{\eta}_{\lessgtr}=\left[\frac{\bar{\Delta}}{2} \pm \sqrt{\frac{\bar{\Delta}^{2}}{4}-\operatorname{det} V}\right]^{1 / 2}
$$

where $\bar{\Delta}=\operatorname{det} A+\operatorname{det} B-2 \operatorname{det} C$. Moreover, inequality (25) can be recast as a constraint $\bar{\Delta} \leq \frac{1}{4}+4 \operatorname{det} V$, leading to $\left(\bar{\eta}_{<}^{2}-\frac{1}{4}\right)\left(\bar{\eta}_{>}^{2}-\frac{1}{4}\right) \geq 0$. Assuming $\bar{\eta}_{>}>\bar{\eta}_{<}$, the PHS separability criterion then simply reads

$$
\bar{\eta}_{<} \geq \frac{1}{2}
$$

Violating the separability criterion, namely $\bar{\eta}_{<}<\frac{1}{2}$, can serve as a measure for the entanglement that we will focus on in this paper. It has also been suggested that negativity [5] and logarithmic negativity [6], which are defined respectively as,

$$
N(\rho)=\max \left\{0, \frac{1-\bar{\eta}_{<}}{2 \bar{\eta}_{<}}\right\}, \quad E(\rho)=\max \left\{0,-\ln 2 \bar{\eta}_{<}\right\}
$$

are good entanglement measures. In particular, they do not increase on average under various local quantum operations. The roles of these entanglement measures in our setup deserve further study.

\section{Numerical/analytical analysis of quantum entanglement}

Violation of the separability criterions, namely $\zeta_{+}<0$ and $\bar{\eta}_{<}<1 / 2$, indicates the existence of quantum entanglement of the systems. We now numerically compute $\zeta_{+}$and $\bar{\eta}_{<}$in (38) and (40) respectively to examine their behaviors, which are shown in Fig. 1. For a given value $\Omega, \sigma\left(\Omega^{2}>\sigma\right), \gamma_{1}$ and $\gamma_{2}$, and also for a fixed $\beta_{2}$, we depict $\zeta_{+}$and $\bar{\eta}_{<}-1 / 2$ as a function of $\beta_{1}$. We find the critical value $\beta_{1 c}$ obtained from $\zeta_{+}=0$ and $\bar{\eta}_{<}=1 / 2$, above which $\left(\beta_{1} \geq \beta_{1 c}\right)$ quantum entanglement exists. In the range of $\beta_{1} \geq \beta_{1 c}$, we see that $\bar{\eta}_{<}$is a monotonically decreasing function of $\beta_{1}$ whereas $\zeta_{+}$is not. It is known that $\bar{\eta}_{<}$can serve as a sensible measure as quantifying the degree of the entanglement through negativity and logarithmic negativity for example. Fig. 2 shows the line of the critical values of $\beta_{1 c}$ and 
$\beta_{2 c}$. In the left-panel we choose $\gamma_{1}=\gamma_{2}$, and in the right-panel, $\gamma_{1}<\gamma_{2}$. In the regime of $\beta_{2 c}>\beta_{1 c}$ and for the same $\beta_{2 c}$, the corresponding $\beta_{1 c}$ given by this critical temperature line in the case of $\gamma_{1}<\gamma_{2}$ (right-panel) changes to a much smaller value than that in the case of $\gamma_{1}=\gamma_{2}$ (left-panel). Nevertheless, for $\beta_{1 c}>\beta_{2 c}$, and for the same $\beta_{1 c}$, the value of $\beta_{2 c}$ in the case of $\gamma_{1}<\gamma_{2}$ (right-panel) becomes larger than that in the case $\gamma_{1}=\gamma_{2}$ (left-panel). Thus, the difference between $\gamma_{1}$ and $\gamma_{2}\left(\gamma_{1}<\gamma_{2}\right)$ can push the critical temperature in the bath $1\left(T_{1 c}=1 / \beta_{1 c}\right)$ to a higher temperature for a fixed $\beta_{2 c}$ in the regime $\beta_{2 c}>\beta_{1 c}$ whereas it also pulls the critical temperature $\left(T_{2 c}=1 / \beta_{2 c}\right)$ in the bath 2 down to a lower value for a fixed $\beta_{1 c}$ in the regime $\beta_{1 c}>\beta_{2 c}$. This is one of our main results in this paper to be further studied analytically in below.

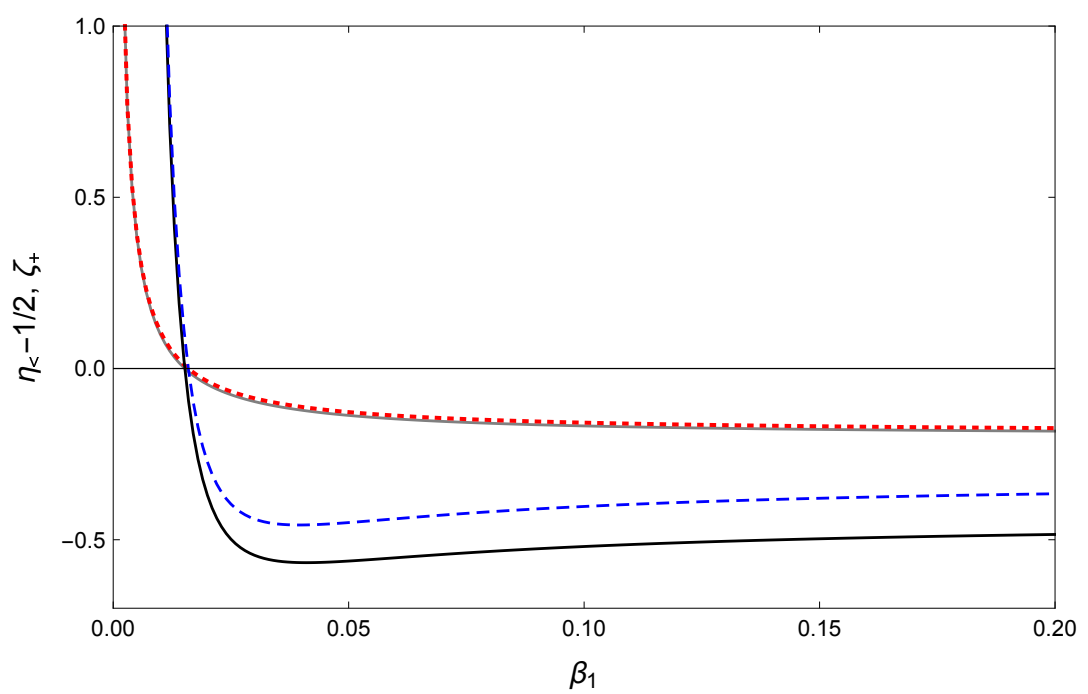

Figure 1: The separability criterions are all dimensionless and depend on dimensional variables given with respect to some relevant unit of, say $\Omega_{0}$. Thus, the criterions $\zeta_{+}$(thick solid line) and $\bar{\eta}_{<}-1 / 2$ (thin solid line) are plotted as the function of $\beta_{1}$ in the unit of $1 / \Omega_{0}$ by fixing $\beta_{2}=1.5 / \Omega_{0}$, and the parameters $\Omega=5 \Omega_{0}$, and $\sigma=24 \Omega_{0}^{2}, \gamma_{1}=0.005 \Omega_{0}$, and $\gamma_{2}=0.25 \Omega_{0}$ according to the exactly numerical results obtained from (38) and (40) respectively in that the momentum integration has logarithmic divergence to be cut off by the chosen cutoff scale $\Lambda=5000 \Omega_{0}$. The approximate results for $\zeta_{+}$(blue dotted line) and $\bar{\eta}_{<}-1 / 2$ (red dotted line) are also plotted by substituting analytically approximate expressions of the covariance matrix elements in (43)-(48) to (38) and (40). We see a good agreement between the exact and approximate results in the parameter regime of validity of the approximations, namely $\beta_{1} \Omega_{+} \ll 1$ and $\beta_{2} \Omega_{-} \gg 1$ with $\Omega_{+} \simeq 7 \Omega_{0}$ and $\Omega_{-} \simeq 1 \Omega_{0}$

In this two oscillators system, two real parts of (30) are $\Omega_{+}$and $\Omega_{-}$with $\Omega_{+}>\Omega_{-}$for $\sigma>0$. To find the analytical expression for the critical temperatures, we approximate the covariance matrix elements by considering the high- $\mathrm{T}$ limit $\beta_{1} \Omega_{+} \ll 1$ with reference to the higher frequency mode in the bath 1 and the low- $\mathrm{T}$ limit $\beta_{2} \Omega_{-} \gg 1$ with reference to the 

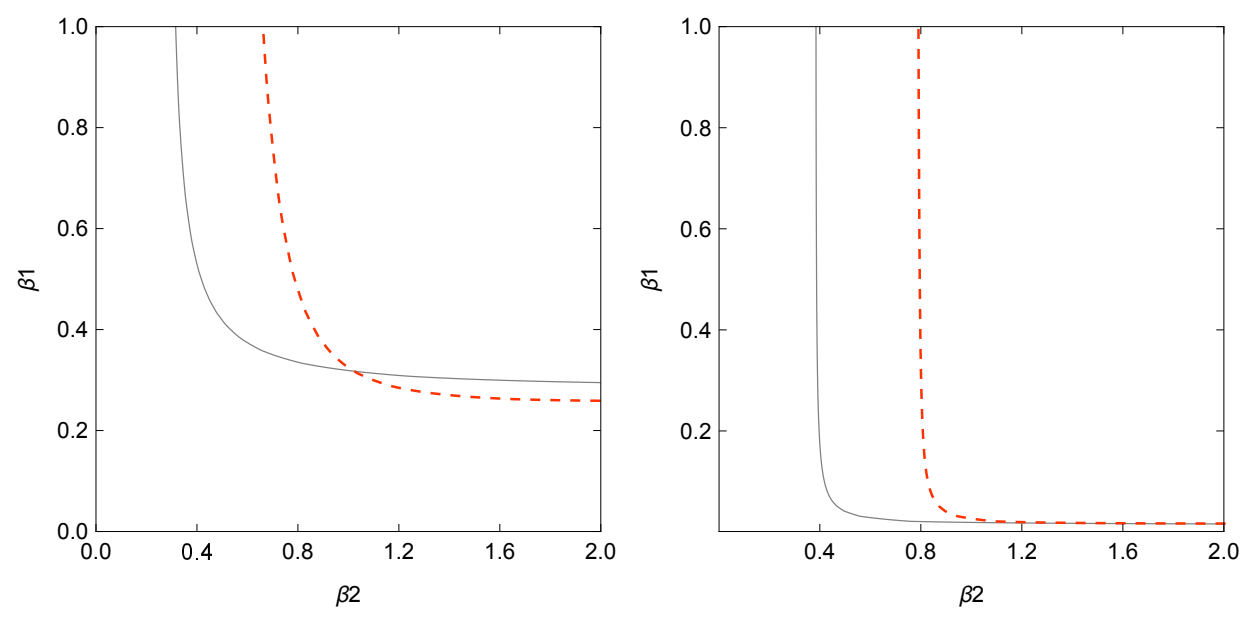

Figure 2: The line of the critical values of $\beta_{1 c}$ and $\beta_{2 c}$ in the unit of $1 / \Omega_{0}$ (black solid line) exactly determined by the sympletic eigenvalue $\eta_{<}=1 / 2$ in (40) and obtained numerically is drawn for $\gamma_{1}=\gamma_{2}=0.25 \Omega_{0}$ (left-panel) and for $\gamma_{1}=0.005 \Omega_{0}, \gamma_{2}=0.25 \Omega_{0}$ (right-panel) as a comparison where the parameters $\Omega$ and $\sigma$ remain the same as in Fig. 1. The critical value line (red dotted line) is also drawn with the same parameters above, but is determined by substituting the approximate covariance matrix elements in (43)-(48) into (40), which also show a good agreement with the exact results in the regime of $\beta_{1} \Omega_{+} \ll 1, \beta_{2} \Omega_{-} \gg 1$ with $\Omega_{+} \simeq 7 \Omega_{0}$ and $\Omega_{-} \simeq 1 \Omega_{0}$

lower frequency mode instead in the bath 2 , and also $\gamma_{a} \ll \Omega_{ \pm}$in the weak damping limit. 
Retaining the terms at the relevant order they are summarized as

$$
\begin{aligned}
& V_{11}=V_{33} \\
& =\frac{1}{m}\left(\frac{\Omega^{2}}{\Omega_{+}^{2} \Omega_{-}^{2}}\right) \frac{\gamma_{1}}{\left(\gamma_{1}+\gamma_{2}\right)} \frac{1}{\beta_{1}}+\frac{\gamma_{2}}{4 m\left(\gamma_{1}+\gamma_{2}\right)}\left(\frac{1}{\Omega_{+}}+\frac{1}{\Omega_{-}}\right)+\frac{\gamma_{2}}{2 \pi m \sigma} \ln \left(\frac{\Omega_{+}}{\Omega_{-}}\right) \\
& -\frac{\gamma_{2}}{2 \pi m}\left(\frac{\Omega^{2}}{\Omega_{+}^{2} \Omega_{-}^{2}}\right)+\frac{2 \pi \gamma_{2} \sigma^{2}}{3 m \Omega_{+}^{4} \Omega_{-}^{4}} \frac{1}{\beta_{2}^{2}}+\mathcal{O}\left(\gamma_{a}^{2}\right) \\
& V_{22}=\frac{\gamma_{1} m}{\pi} \ln \left(\beta_{1} \Lambda\right) \Theta\left(\beta_{1} \Lambda-1\right)+\frac{m \gamma_{1}}{\left(\gamma_{1}+\gamma_{2}\right)} \frac{1}{\beta_{1}}+\frac{m \gamma_{2}}{4\left(\gamma_{1}+\gamma_{2}\right)}\left(\Omega_{+}+\Omega_{-}\right)-\frac{m \gamma_{2}}{2 \pi} \\
& +\frac{m \gamma_{2} \Omega^{2}}{2 \pi \sigma} \ln \left(\frac{\Omega_{+}}{\Omega_{-}}\right)+\frac{4 \pi^{3} m \gamma_{2} \sigma^{2}}{15 \Omega_{+}^{4} \Omega_{-}^{4}} \frac{1}{\beta_{2}^{4}}+\mathcal{O}\left(\gamma_{a}^{2}\right) \\
& V_{44}=\frac{m \gamma_{1}}{\pi} \ln \left(\beta_{1} \Lambda\right) \Theta\left(\beta_{1} \Lambda-1\right)+\frac{m \gamma_{1}}{\left(\gamma_{1}+\gamma_{2}\right)} \frac{1}{\beta_{1}}+\frac{2 m \gamma_{2}}{\pi} \ln \left(\frac{\Lambda}{\Omega_{+} \Omega_{-}}\right)+\frac{m \gamma_{2}}{4\left(\gamma_{1}+\gamma_{2}\right)}\left(\Omega_{+}+\Omega_{-}\right) \\
& +\frac{m \gamma_{2} \Omega^{2}}{2 \pi \sigma} \ln \left(\frac{\Omega_{+}}{\Omega_{-}}\right)-\frac{m \gamma_{2}}{2 \pi}+\frac{4 \pi^{3} m \gamma_{2} \sigma^{2}}{15 \Omega_{+}^{4} \Omega_{-}^{4}} \frac{1}{\beta_{2}^{4}}+\mathcal{O}\left(\gamma_{a}^{2}\right) \\
& V_{13}=-\frac{1}{m}\left(\frac{\sigma}{\Omega_{+}^{2} \Omega_{-}^{2}}\right) \frac{\gamma_{1}}{\left(\gamma_{1}+\gamma_{2}\right)} \frac{1}{\beta_{1}}+\frac{\gamma_{2}}{4 m\left(\gamma_{1}+\gamma_{2}\right)}\left(\frac{1}{\Omega_{+}}-\frac{1}{\Omega_{-}}\right)+\frac{\gamma_{2}}{2 \pi m}\left(\frac{\sigma}{\Omega_{+}^{2} \Omega_{-}^{2}}\right) \\
& -\frac{2 \pi \gamma_{2} \sigma \Omega^{2}}{3 m \Omega_{+}^{4} \Omega_{-}^{4}} \frac{1}{\beta_{2}^{2}}+\mathcal{O}\left(\gamma_{a}^{2}\right) \\
& V_{14}=-\left(\frac{2}{\sigma}\right) \frac{\gamma_{1} \gamma_{2}}{\left(\gamma_{1}+\gamma_{2}\right)} \frac{1}{\beta_{1}}+\frac{8 \pi^{3} \gamma_{1} \gamma_{2} \sigma}{15 \Omega_{+}^{4} \Omega_{-}^{4}} \frac{1}{\beta_{2}^{4}}+\mathcal{O}\left(\gamma_{a}^{3}\right) \\
& V_{24}=\left(\frac{m \sigma}{12}\right) \frac{\gamma_{1} \beta_{1}}{\gamma_{1}+\gamma_{2}}+\frac{m \gamma_{2}}{4\left(\gamma_{1}+\gamma_{2}\right)}\left(\Omega_{+}-\Omega_{-}\right)-\frac{m \gamma_{2}}{2 \pi} \ln \left(\frac{\Omega_{+}}{\Omega_{-}}\right)-\frac{4 \pi^{3} m \gamma_{2} \sigma \Omega^{2}}{15 \Omega_{+}^{4} \Omega_{-}^{4}} \frac{1}{\beta_{2}^{4}}+\mathcal{O}\left(\gamma_{a}^{2}\right) .
\end{aligned}
$$

Substituting the analytical approximate expressions to (38) and (40), we can then compare the approximate results of $\zeta_{+}$and $\bar{\eta}_{<}$with their exact numerical ones as shown in Fig. 1, where the critical value of $\beta_{1 c}$ can be correctly obtained in the case of $\beta_{1 c} \Omega_{-} \ll 1$ by choosing $\beta_{2 c} \Omega_{+} \gg 1$ within the parameter regime where the approximate expressions are valid. The line of the critical values, $\beta_{1 c}$ and $\beta_{2 c}$, resulting from the approximate results are also shown in Fig. 2, which provide a good analytical estimate on $\beta_{1 c}$ and $\beta_{2 c}$ again in the regime of $\beta_{1 c} \Omega_{+} \ll 1, \beta_{2 c} \Omega_{-} \gg 1$. Substituting (43)-(48) into (38) and (40), the condition $\bar{\eta}_{<}=1 / 2$ in the PHS criterion determines the line of the critical values analytically. To obtain the analytical expression for the critical temperatures needs further approximations. Considering the temperature-dependent parts in (43)-(48), in the case $\beta_{1 c} \Omega_{+} \ll 1, \beta_{2 c} \Omega_{-} \gg 1$, the terms of $1 / \beta_{2}^{2}, 1 / \beta_{2}^{4}$, and $\beta_{1}$ are relatively small and thus can be safely dropped out. Thus, we consider the $1 / \beta_{1}$ dependence only. As for the temperature-independent parts, in the limits of $\gamma_{1} \ll \gamma_{2}$ and $\gamma_{1}, \gamma_{2} \ll \Omega_{ \pm}$, the terms proportional to $\gamma$ are ignorably small except for the 
cut-off dependent contributions where the cutoff scale is chosen to be $\Lambda \gg \Omega$. The PHS criterion then gives

$$
\begin{aligned}
\beta_{1 c} \simeq & 4 \pi \gamma_{1} /\left\{\left[\pi^{2}\left(4 \gamma_{1}^{2} \Omega_{+}^{2}+8 \gamma_{1} \gamma_{2} \Omega_{+}^{2}+\gamma_{2}^{2}\left(5 \Omega_{+}^{2}-2 \Omega_{+} \Omega_{-}+\Omega_{-}^{2}\right)\right)+\right.\right. \\
& \left.\gamma_{2}^{2}\left(\gamma_{1}+\gamma_{2}\right) \ln \left|\Lambda^{4} / \Omega_{-}^{4}\right|\left(2 \pi\left(\Omega_{-}-\Omega_{+}\right)+\left(\gamma_{1}+\gamma_{2}\right) \ln \left|\Lambda^{4} / \Omega_{-}^{4}\right|\right)\right]^{1 / 2} \\
& \left.-\gamma_{2}\left(\gamma_{1}+\gamma_{2}\right) \ln \left|\Lambda^{4} / \Omega_{-}^{4}\right|-\pi \gamma_{2}\left(\Omega_{+}+\Omega_{-}\right)\right\}, \\
\simeq & \frac{4 \pi \gamma_{1}}{\gamma_{2}\left[\pi \sqrt{5 \Omega_{+}^{2}-2 \Omega_{+} \Omega_{-}+\Omega_{-}^{2}}-\pi\left(\Omega_{+}+\Omega_{-}\right)-\gamma_{2} \ln \left|\Lambda^{4} / \Omega_{-}^{4}\right|\right]}+\mathcal{O}\left(\gamma_{1}^{2}\right) .
\end{aligned}
$$

Also, notice that to be consistent with the small $\gamma$ approximation, the choice of the cutoff scale is such that the contributions from the $\ln \Lambda$ dependence are sub-leading as compared with the leading order terms given by $\Omega$ and $\sigma$ in (49). The solution of $\beta_{1 c}$ is found to be $\beta_{1 c} \propto \gamma_{1} / \gamma_{2}$ for $\gamma_{1}<\gamma_{2}$ where $\beta_{1 c}$ is significantly suppressed by the smallness of $\gamma_{1}$ so that the associated critical temperature $T_{1 c}=1 / \beta_{1 c}$ is boosted into the hight- $T$ regime with $T_{1 c} / \Omega_{+} \gg 1$ with respect to the higher frequency $\Omega_{+}$of the normal mode. On the contrary, in the regime $\beta_{1 c}>\beta_{2 c}$, although in Fig. $2, \beta_{2 c}$ is not within high- $T$ regime, roughly speaking we expect $\beta_{2 c}$ is given by (49) by the replacement of $\gamma_{1} \leftrightarrow \gamma_{2}$ for $\beta_{1 c}$ being a large value. Thus, for the same $\beta_{1 c}, \beta_{2 c}$ increases as $\gamma_{1}$ decreases from $\gamma_{1}=\gamma_{2}$ to $\gamma_{1}<\gamma_{2}$ with a fixed $\gamma_{2}$ being consistent with the numerical results shown in Fig. 2. In the case of $\gamma_{1}=\gamma_{2}$, both $\beta_{1 c}$ and $\beta_{2 c} \propto \mathcal{O}\left(1 / \Omega_{+}\right)$reduces to the findings in [21]. Although the effects from the different damping parameters $\gamma_{1} \neq \gamma_{2}$ can push one of the critical temperatures to a higher one, it may not be so robust to boost the critical temperatures in both baths to the one higher than the oscillator frequency as in the case via parametric driving due to the timedependent mutual interaction between two oscillators. Our results are consistent with the main conclusion in [22] that the large temperature difference in $T_{1}=1 / \beta_{1}$ and $T_{2}=1 / \beta_{2}$ in the case of nondegenerate normal modes $\left(\Omega_{+} \neq \Omega_{-}\right)$can produce the nonvanishing steadystate coherence in high- $T$ limits. And we furthermore show the line of the critical values of $T_{1 c}$ and $T_{2 c}$ below which the entanglement can survive. Here we carry out more complete calculations with as few approximation as possible, with which to explore how to achieve hot entanglement between two oscillators.

\section{Comparison to the systems with respective temperature-dependent damping parameters from heat baths}

In this section we consider the damping parameter that has the temperature dependence, say $\gamma_{a T}=\bar{\gamma}_{a} T^{\alpha}$ with $\alpha>0$, resulting from the fact that the system-bath coupling term is beyond the linear dependence of the bath's variable. In [31, 32, 33], we study the nonequilibrium dynamics of the mirror with perfect reflection moving in a quantum field. The force acting 

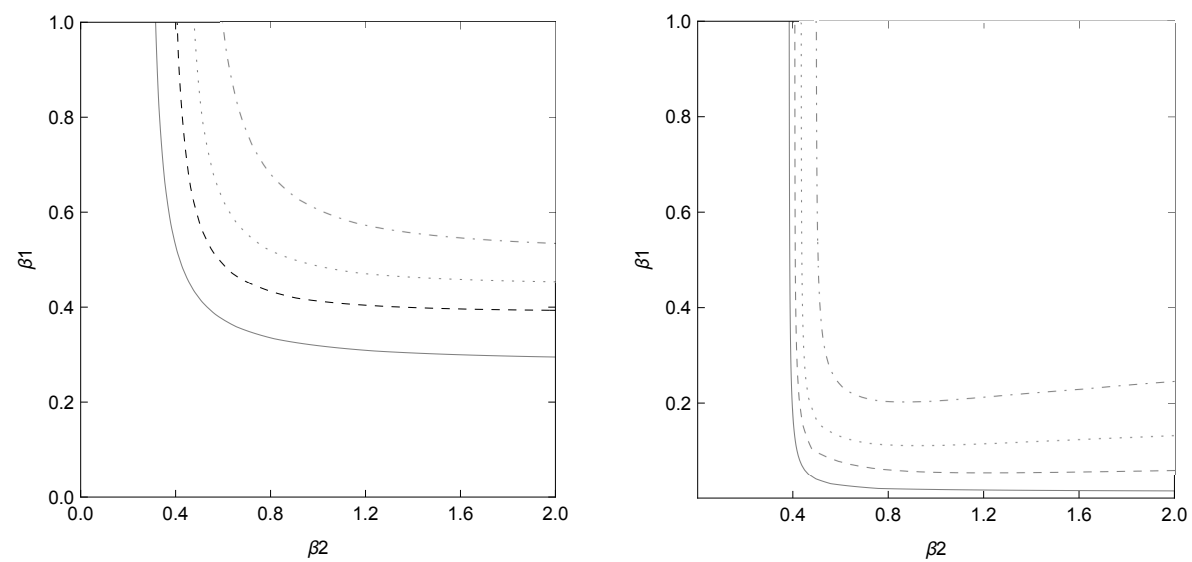

Figure 3: The line of the critical values of $\beta_{1 c}$ and $\beta_{2 c}$ in the unit of $1 / \Omega_{0}$ exactly determined by $\eta_{<}=1 / 2$ in (40) with $\alpha=0$ (solid), $\alpha=1 / 2$ (dashed), $\alpha=1$ (dotted) and $\alpha=2$ (dash-dotted). The values of $\bar{\gamma}_{a}$ are chosen to be $\bar{\gamma}_{1 T}=\bar{\gamma}_{2 T}=0.25 \Omega_{0}^{1-\alpha}$ (left-panel) and $\bar{\gamma}_{1 T}=0.005 \Omega_{0}^{1-\alpha}, \bar{\gamma}_{2 T}=0.25 \Omega_{0}^{1-\alpha}$ (right-panel). The parameters $\Omega$ and $\sigma$ remain the same as in Fig. 1.

on the mirror is the radiation pressure of the environmental field given by the area integral of the stress tensor, which is quadratic in field variables. For the system of the 2-dimensional mirror influenced by a heat bath of free relativistic scalar fields, the temperature-dependent damping parameters are obtained giving $\alpha=4$. Later, our studies are extended to the case of $n$-dimensional mirrors interacting with a heat bath of Lifshitz scalar fields with a dynamic exponent $z$, which are of strongly coupled quantum fields. The corresponding $\alpha$ using the holographic approach can be computed to be $\alpha=(n+2) / z$. Taking the strongly self-coupling effects into account, the value of $\alpha$ can even be a fractional number. With the direct replacement of $\gamma_{a} \rightarrow \gamma_{a T}=\bar{\gamma}_{a} T_{a}^{\alpha}$ in the Hadamard function $G_{H, T_{a}}$ (17) and the matrix $\tilde{D}$ (26), we can numerically calculate the elements of the matrix $V$ from (31)-(37). Then, from them the line of the critical values of $\beta_{1 c}$ and $\beta_{2 c}$ can be determined by the criterion $\bar{\eta}_{<}=1 / 2$ in (40), which are shown in Fig.(3) by choosing the different values of $\alpha$. In the leftpanel (right-panel) of the figure, $\bar{\gamma}_{1}=\bar{\gamma}_{2}\left(\bar{\gamma}_{1}<\bar{\gamma}_{2}\right)$ is chosen together with the same values of $\Omega_{0}$ and $\sigma$ as in Fig.(1). As $\alpha$ increases while keeping the same $\beta_{2 c}$, the corresponding $\beta_{1 c}$ increases. This feature can be understood qualitatively from (49) for $\beta_{2 c} \Omega_{-} \gg 1$, although the values of $\beta_{1 c}\left(\beta_{1 c} \Omega_{+} \geq 1\right)$ are beyond the validity of the approximations to achieve it. With $\gamma_{2 T}=\bar{\gamma}_{2} / \beta_{2}^{\alpha}$, as $\alpha(\alpha>0)$ increases, for $\beta_{2 c} \Omega_{-} \gg 1 \gamma_{2 T}$ becomes smaller, leading to the larger values of $\beta_{1 c}$. In particular, for $\bar{\gamma}_{2}>\bar{\gamma}_{1}$, the critical temperature $T_{1 c}$ can be boosted into a higher value seen in the right-panel of the figure, which can be qualitatively understandable also from (49) with $\beta_{1 c} \propto\left(\bar{\gamma}_{1} / \bar{\gamma}_{2}\right)^{\frac{1}{1+\alpha}}$. Even for the different $\alpha_{a}$, namely $\alpha_{1} \neq \alpha_{2}$ for the bath 1 and the bath $2, \beta_{1 c} \propto\left(\bar{\gamma}_{1} / \bar{\gamma}_{2}\right)^{\frac{1}{1+\alpha_{1}}}$. Thus, the suppression of $\beta_{1 c}$ is the most significant when $\alpha=0$ also seen in the figure. Similar behavior of $\beta_{2 c}$ for the same $\beta_{1 c}$ can also be interpreted from (49) by the exchange of the subscripts $1 \leftrightarrow 2$. Thus, 
in this model, we find that the systems, which couple to heat baths with the temperature independent damping parameters, are more probable to establish hot entanglement by the mutual interaction as compared with the situations with temperature-dependent damping parameters.

\section{Summaries and looking ahead}

The main goal of this work is to study the entanglement of quantum systems at finite temperature. We reconsider the model of two quantum oscillators coupled to its own environment fields through the coupling linear in the field variable at different temperatures $T_{1}$ and $T_{2}$. By tracing out the environmental degrees of freedom exactly, the stochastic effective action is obtained, from which the Heisenberg-Langevin equations for two oscillators are derived including the damping effects of the ohmic form with the general damping parameters, $\gamma_{1} \neq \gamma_{2}$ as well as the noise terms where they obey the fluctuation-dissipation relations. Solving the equations for the general solutions allows us to compute the position and the momentum uncertainties as well as the expectation values of position-momentum cross correlations of the system with the given initially separable Gaussian states of two oscillators. The interaction between two oscillators start to build up the entanglement between them. In the weak damping limit, the late-time behavior of the system is reached by the nonequilibrium steady state. The separability criterions constructed by the covariance matrix elements are computed numerically and analytically. Violation of the criterions implies the existence of the entanglement where the line of the critical temperature can be determined. Considering $\gamma_{1}<\gamma_{2}$, the critical temperature of $T_{1 c}$ can be as high as $T_{1 c} / \Omega_{+} \propto \gamma_{2} / \gamma_{1}$ with reference to the higher frequency of the normal mode. So, we can have $T_{1 c} \gg \Omega_{+}$particularly for $\gamma_{1} \ll \gamma_{2}$, while keeping $T_{2 c} \propto \Omega_{+}$. This interesting finding can be compared with the case of $\gamma_{1}=\gamma_{2}$ where both $T_{1 c}$ and $T_{2 c}$ are found to be the order of $\Omega_{+}$. Thus, the effects from the different damping parameters $\gamma_{1} \neq \gamma_{2}$ can push one of the critical temperatures to a higher one. This might give the possibility of hot entanglement. Nevertheless, this may not be so effective to boost the critical temperatures for both baths as in the case via parametric driving due to the time-dependent interaction between two oscillators. Also we consider the systems with the temperature-dependent damping parameters of the form $\gamma_{a T}=\bar{\gamma}_{a} T^{\alpha}(\alpha>0)$, which can obtained by coupling to environment fields at finite temperature via the area integral of the stress tensor of the field variables. We find that the boost of the critical temperature to a higher value is more noticeable in the case of $\alpha=0$. These findings deserve further experimental justification. Finally, it might deserve a further study on parametrically driven, dissipative harmonic oscillators with different damping parameters for more exploration on hot entanglement, as an extension of the work in [17]. 


\section{Acknowledgments}

This work was supported in part by the Ministry of Science and Technology, Taiwan. We are so grateful to Jen-Tsung Hsiang for illuminating discussions.

\section{References}

[1] Peres A 1996 Phys. Rev. Lett. 77(8) 1413-1415

[2] Horodecki M, Horodecki P and Horodecki R 1996 Physics Letters A 2231 - 8 ISSN 0375-9601

[3] Horodecki P 1997 Physics Letters A 232333 - 339 ISSN 0375-9601

[4] Simon R 2000 Phys. Rev. Lett. 84(12) 2726-2729

[5] Vidal G and Werner R F 2002 Phys. Rev. A 65(3) 032314

[6] Plenio M B 2005 Phys. Rev. Lett. 95(9) 090503

[7] Eisert J and Plenio M B 1999 Journal of Modern Optics 46 145-154

[8] Virmani S and Plenio M 2000 Physics Letters A 26831 - 34 ISSN 0375-9601

[9] Feynman R and Vernon F 2000 Annals of Physics 281547 - 607 ISSN 0003-4916

[10] Caldeira A and Leggett A 1983 Annals of Physics 149374 - 456 ISSN 0003-4916

[11] Caldeira A O and Leggett A J 1981 Phys. Rev. Lett. 46(4) 211-214

[12] Caldeira A and Leggett A 1983 Physica A: Statistical Mechanics and its Applications 121587 - 616 ISSN 0378-4371

[13] Gröblacher S, Trubarov A, Prigge N, Cole G D, Aspelmeyer M and Eisert J 2015 Nature Communications 67606

[14] Thompson J D, Zwickl B M, Jayich A M, Marquardt F, Girvin S M and Harris J G E 2008 Nature 452 $72-75$

[15] Gröblacher S, Hertzberg J B, Vanner M R, Cole G D, Gigan S, Schwab K C and Aspelmeyer M 2009 Nature Physics 5 485-488

[16] Naik A, Buu O, LaHaye M D, Armour A D, Clerk A A, Blencowe M P and Schwab K C 2006 Nature 443 193-196

[17] Galve F, Pachón L A and Zueco D 2010 Phys. Rev. Lett. 105(18) 180501

[18] Estrada A F and Pachón L A 2015 New Journal of Physics 17033038

[19] Vedral V 2010 Nature 468 769-770

[20] Hsiang J T and Hu B 2015 Physics Letters B 750396 - 400 ISSN 0370-2693

[21] Hsiang J T and Hu B L 2015 Journal of High Energy Physics 201590

[22] Boyanovsky D and Jasnow D 2017 Phys. Rev. A 96(1) 012103

[23] Fong K Y, Li H K, Zhao R, Yang S, Wang Y and Zhang X 2019 Nature 576 243-247

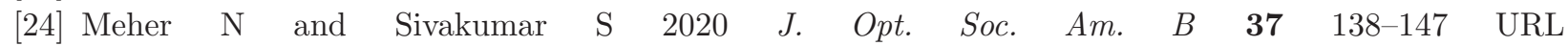
http://josab.osa.org/abstract.cfm?URI=josab-37-1-138

[25] Biehs S A and Agarwal G S 2013 J. Opt. Soc. Am. B $\quad 30$ 700-707 URL http://josab.osa.org/abstract.cfm?URI=josab-30-3-700

[26] Xuereb A, Imparato A and Dantan A 2015 New Journal of Physics 17055013 URL https://doi.org/10.1088/1367-2630/17/5/055013

[27] Asadian A, Manzano D, Tiersch M and Briegel H J 2013 Phys. Rev. E 87(1) 012109 URL https://link.aps.org/doi/10.1103/PhysRevE.87.012109

[28] Hsiang J T and Hu B 2015 Annals of Physics 362139 - 169 ISSN 0003-4916

[29] Simon R, Sudarshan E C G and Mukunda N 1987 Phys. Rev. A 36(8) 3868-3880

[30] Simon R, Mukunda N and Dutta B 1994 Phys. Rev. A 49(3) 1567-1583

[31] Wu C H and Lee D S 2005 Phys. Rev. D 71(12) 125005

[32] Yeh C P, Hsiang J T and Lee D S 2014 Phys. Rev. D 89(6) 066007

[33] Lee D S and Yeh C P 2019 Journal of High Energy Physics 201968 